\title{
Aplicación práctica de internet de las cosas: seguridad de casa habitación
}

\author{
Practical application of internet of things: home security \\ SÁNCHEZ-DELGADO, Octaviơ $†$, NOGUERON-SOTO, Alfonso, ALFARO-HERRERA, Julio César \\ y HERNÁNDEZ-GUZMÁN Julieta Edith
}

Universidad Tecnológica de Tehuacán, Prolongación de la 1 sur 1001 San Pablo Tepetzingo, Tehuacán Puebla C.P 75859.

ID $1^{\text {er }}$ Autor: Octavio, Sánchez-Delgado / ORC ID: 0000-0002-7073-0522 Researcher ID: A-8203-2019, CVU: 957303

ID $1^{\text {er }}$ Coautor: Alfonso, Nogueron-Soto / ORC ID: 0000-0002-2306-327X Publons: 3092386, CVU: 790425

ID $2^{\text {do }}$ Coautor: Christian Galicia-Garcia / ORC ID: 0000-0001-7796-4295 Researcher ID: X-6362-2018, CVU: 618470

ID $3^{\text {er }}$ Coautor: Julieta Edith, Hernández-Guzmán / ORC ID: 0000-0002-3166-987X Publons: 3095819, CVU: 1014010

DOI: $10.35429 / J A D \cdot 2019.7 .3 .1 .10$

Recibido 10 de Enero, 2019; Aceptado 25 de Marzo, 2019

\begin{abstract}
Resumen
En los primeros meses del 2019, en algunas regiones de México se ha notado un incremento en el robo a casa habitación, como es el caso del municipio de Tehuacán en Puebla. Es entonces que se genera una alternativa que implementa el Internet de las Cosas con el objetivo de reducir el índice de robos a casa habitación, se genera un sistema que controla la apertura de puertas y la activación de alarmas en caso de apertura indebida, con ello se busca beneficiar a las personas de Tehuacán y del país. El sistema de seguridad con internet de las cosas permite al usuario gestionar y controlar el acceso a las puertas del hogar, el sistema funciona gracias a una aplicación que se instala en un teléfono inteligente, que a su vez tendrá una conexión con los dispositivos que estarán instalados en las puertas, de esta manera solo el usuario con la aplicación y una clave podrá abrir las puertas de forma segura, en caso contrario se dispara una alarma que pone en alerta al dueño de la casa donde quiera que esté y puede llamar a los números de emergencia para evitar ser víctima de un delito.
\end{abstract}

Internet de las Cosas, Robo a Casa habitación, Seguridad

\begin{abstract}
In the first months of 2019, in some regions of Mexico there has been an increase in home theft, as is the case of the municipality of Tehuacán in Puebla. It is then that an alternative is generated that implements the Internet of Things with the aim of reducing the rate of home theft, a system is generated that controls the opening of doors and the activation of alarms in case of undue opening, with it It seeks to benefit the people of Tehuacán and the country. The security system with internet of things allows the user to manage and control access to the doors of the home, the system works thanks to an application that is installed on a smartphone, which in turn will have a connection with the devices that will be installed in the doors, in this way only the user with the application and a key can open the doors safely, otherwise an alarm is triggered that alerts the owner of the house wherever he is and can call emergency numbers to avoid being a victim of a crime.
\end{abstract}

Internet of Things, Home Theft, Security 


\section{Introducción}

Aunque las autoridades ponen su mayor empeño en mejorar muchos aspectos de seguridad pública, la realidad es muy diferente, en agosto de 2018 en México se contaban con 0.8 policías por cada 1000 habitantes, aspecto que incumple el estándar de la Organización de las Naciones Unidas (ONU), esto según el portal de noticias excelsior.com.mx. Para agosto de 2019 se tiene un incremento de 1.0 policías por cada 1000 habitantes, lo cual sigue sin cumplir el estándar de la ONU, que según el portal de noticias heraldodemexico.com.mx es de 3.0 policías por cada 1000 habitantes. Con estos datos se comprueba que aún es insuficiente el trabajo que realizan las autoridades en materia de seguridad pública.

Con la ola de inseguridad que se ha desencadenado en la actualidad, hay delitos que requieren de mucha atención, en este caso se habla del robo a casa habitación ya que va en crecimiento en algunas zonas del país, las autoridades dan recomendaciones para evitar este delito, ya que dejar la luz encendida ya no es una buena opción para ahuyentar a los ladrones, es por ello que es necesario tomar cartas en el asunto.

En la ciudad de Tehuacán. Según la página oficial "diarioelmundo.com.mx" en el primer trimestre del 2019 se duplicaron los robos a casa habitación en Tehuacán, siendo el año con más casos registrados desde el 2015. En los últimos meses el delito a viviendas ha ido al alza, pues hace cinco años solo se contabilizaron 20 robos en el primer trimestre del año y logró disminuir a 15 en el 2018, no obstante, para este año se han iniciado 30 carpetas de investigación de esta índole en el primer trimestre del año, lo cual convierte al 2019 en el año con más robos a casa habitación, por ello es urgente mejorar el plan de seguridad.

Toda esta inseguridad ha provocado gran incertidumbre entre la ciudadanía, nadie está a salvo de este y otros delitos. Con esa inquietud se despierta el interés para implementar un sistema que ayude a disminuir el delito en este ámbito. Aprovechar la tecnología y las herramientas para implementar métodos que alerten a tiempo a los dueños de la casa y a las autoridades para atrapar en fragancia a los delincuentes.

\section{Objetivos}

Crear un sistema de seguridad antirrobo que sea capaz de asegurar el cierre de puertas de los inmuebles, aplicando tecnología de internet de las cosas para detectar el forcejeo de chapas y controlar la apertura de puertas, para reducir el índice de robos a hogares al alertar con notificaciones en tiempo real a los dueños de la casa habitación sobre los sucesos del acceso principal de su casa habitación.

\section{Objetivos específicos}

Generar un sistema electrónico que tome la función de chapa, controlado por programación

Crear un sistema para el acceso a su hogar con historial de aperturas y que administre usuarios y contraseñas de acceso

Crear una aplicación móvil que tome el rol de llave para la apertura de la puerta.

Crear una alarma en tiempo real que alerte al dueño de la casa sobre incidentes.

\section{Marco Teórico}

A continuación, se enlistan y definen los principales temas tratados durante el desarrollo de este trabajo.

Seguridad:

Se considera que la seguridad es una sensación o sentimiento de total confianza frente a peligros externos que afecten al individuo y/o a sus pertenencias.

\section{Sistema de Seguridad:}

Un Sistema de seguridad es un conjunto de elementos e instalaciones interrelacionados que tienen como objetivo establecer un nivel de protección a las personas y bienes materiales establecidos en un lugar determinado, la protección se da frente a sucesos como robo, atraco, incendio, daño físico, etc.

Tipos de Sistemas de Seguridad: 
Los sistemas de seguridad se clasifican en 4 bloques como son: Robo y atraco, Incendio, Anti-hurto y Especiales, en este documento se plasma el trabajo de un sistema de seguridad de robo y atraco, los cuales involucran sensores y sistemas de alarma, avisos a centrales de seguridad, dispositivos de acceso, etc.

Elementos de un Sistema de Seguridad:

En la Figura 1 se muestra el esquema de sistema de seguridad tradicional, por ejemplo, una sirena, unidad de control y los sensores ya forman un sistema de seguridad básico.

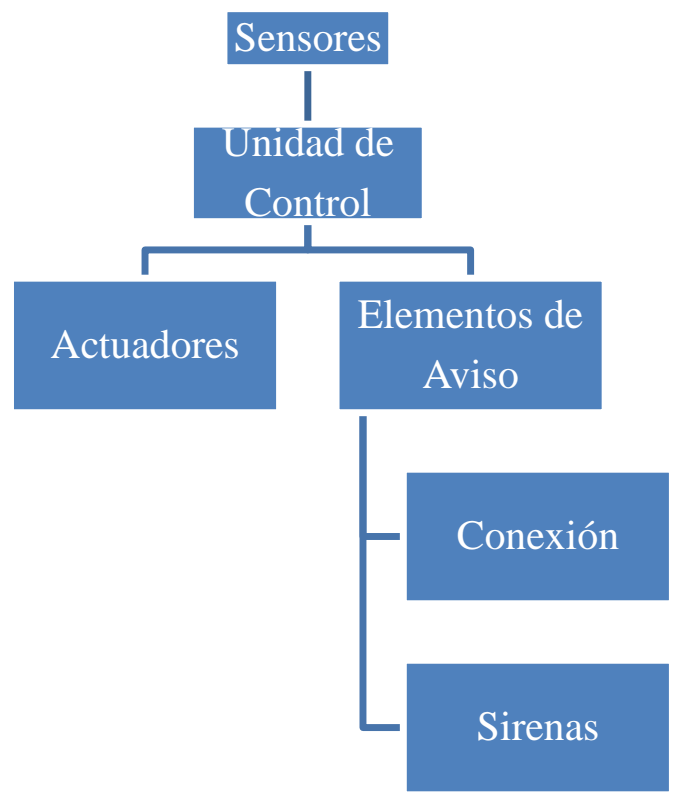

Figura 1 Sistema de Seguridad

Fuente: elaboración propia con información de gruponavarro.pe

\section{Sensor:}

Se refiere a un elemento de medición que detecta una señal del ambiente real y lo transforma en una señal que es comprendida y puede ser procesada por un sistema, un ejemplo claro es el sensor ultrasónico, el cual permite conocer la distancia en centímetros de la ubicación de objetos o personas.

El cual envía pulsos haciendo hondas que rebotan en una superficie.

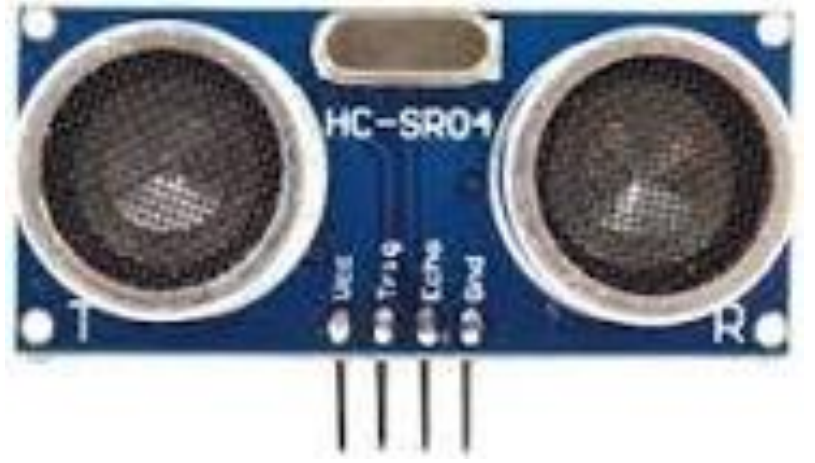

Figura 2 Sensor Ultrasónico

Fuente: tomado de carrod.mx

Actuadores: los actuadores son dispositivos que permiten utilizar distintos tipos de energía para generar un movimiento o cambio de estado dentro de un sistema automatizado, algunos actuadores aplican fuerzas neumáticas, hidráulicas o eléctricas, siendo este ultimo el utilizado el presente trabajo. Un servo motor trasforma energía eléctrica e mecánica para, en este caso abrir o cerrar una puerta.

Unidades de control: es el dispositivo que regula cada uno de los movimientos de los actuadores, realiza acciones de cálculo, obtiene y procesa información de los sensores, envía y recibe señales a otros dispositivos, es programable y genera información útil para la toma de decisiones.

Arduino: es una plataforma electrónica de hardware y software fácil de utilizar, se basa en una placa con un micro controlador y un entorno de desarrollo con el cual se realizan tareas esenciales de un controlador como recibir señales analógicas y digitales, acciones de cálculo, etc.

Conexiones: son el medio por el cual interactúan uno o más dispositivos, con un controlador, en el caso de los sensores generan una conexión Dispositivo a Dispositivo generalmente de manera cableada a corta distancia, así mismo el dispositivo de puede comunicar con un servidor de información, a esta se le denomina Dispositivo a Maquina y puede ser por conexiones físicas como el cable Ethernet, o puede ser por medios inalámbricos como el estándar WI-FI o Bluetooth. 
Internet de las Cosas: por sus siglas en inglés IoT, es un sistema de dispositivos interrelacionados, maquinas mecánicas y digitales, objetos animales o personas que tienen la capacidad de enviar datos a través de una red sin la intervención directa del hombre.

Lenguaje de Programación: es un idioma artificial diseñado para expresar operaciones que son realizadas por computadoras, micro controladores y equipos especializados. El lenguaje de programación es la forma en que el humano da indicaciones a las computadoras, el humano utiliza lenguaje que reconoce completamente, mientras que la computadora solo entiende $0 \mathrm{~s}$ y $1 \mathrm{~s}$.

JavaScript: es un lenguaje de programación que se utiliza principalmente para crear paginas Web dinámicas. JavaScript permite desarrollar sitios WEB, video juegos, aplicaciones de escritorio, , domótica y más, es importante resaltar que Java (otro lenguaje de programación) no es igual JavaScript.

Node.js: es un entorno que trabaja en tiempo de ejecución, de código abierto, multiplataforma, que permite a los desarrolladores crear toda clase de herramientas del lado del servidor y aplicaciones en JavaScript. Su principal ventaja es que utiliza a JavaScript como lenguaje de programación lo cual facilita su aprendizaje.

OneSignal: es una plataforma basada en JavaScript que permite el envío de Notificaciones Push a través de diferentes plataformas que estén debidamente registradas, una vez que el dispositivo esta registrado ha quedado listo para el envío de notificaciones desde un panel de control.

Socket.IO: es una librería que permite manejar eventos en tiempo real mediante una conexión TCP y utiliza el lenguaje de programación JavaScript, es multiplataforma, envía texto, audio y video, esta centrado en la velocidad de respuesta por lo que se le conoce como Realtime.
Angular: es un framework para aplicaciones WEB en TypeScript, es de código abierto, angular se utiliza para crear y mantener aplicaciones web de una sola página, es compatible con todos los navegadores de internet, se crean módulos y se agregan según se requiera y es multiplataforma, lo que facilita el diseño de código.

ReactNative: es una librería de Javascritp enfocada en el diseño y desarrollo de interfaces de usuario, es la base para construir cualquier cosa utilizando JavaScript, facilita el desarrollo de código, debido a que ya cuenta con fragmentos de código y complementos ya listos para utilizarse. Es por ello que su principal aplicación es el desarrollo de aplicaciones WEB, mejor ordenadas y con menos líneas de código.

Electron: es una librería desarrollada para construir aplicaciones multiplataforma con con HTML, CSS y JavaScript, realiza una combinación de herramientas como Chromium y Node.js, lo que permite implementar funciones nativas, herramientas de debugging y permite generar archivos ejecutables que son compatibles con Windows, Linux y Mac OS. El framework es apoyada por la comunidad GitHub.

Firebase: es una base de datos NoSQL alojada en la nube, su principal función es que sincroniza los datos con todos los clientes en tiempo real y estos se mantienen disponibles cuando la Aplicación móvil no de encuentra disponible. Los datos se almacenan en formato JSON, su uso experimental es gratuito con poca demanda de conexión, si se requiere un soporte para mas información, es necesario generar un espacio de pago mensual.

ESP8266: es un puente de puerto Serial a Wifi, que tiene un controlador para el manejo del protocolo TCP/IP y el software necesario para la conexión 802.11, el cual brinda un complemento a cualquier microcontrolador y coloca a un paso el desarrollo del Internet de las cosas. 


\section{Metodología}

Diseño de Electrónica.

El primer paso para la creación de la propuesta, consistió en el diseño y desarrollo del prototipo electrónico, el cual consiste en un Actuador, un sensor y un controlador, para la prueba inicial se utilizan un servomotor SG90 y un led, un sensor ultrasónico HC-SR04 y 2 botones conectados a un Arduino UNO, este último será el controlador del sistema. El servomotor SG90 será utilizado para accionar el cerrojo de la puerta con lo cual se permite la apertura de la misma; al momento de cerrar la puerta se deberá accionar nuevamente el actuador, pero en sentido contrario para que la puerta se mantenga cerrada, el led es un indicativo visual, el cuan encenderá cuando se tenga la puerta abierta, esto a manera de que el controlador muestre el estado de la puerta de acuerdo a sus parámetros.

Se considera que la puerta no tendrá una chapa tradicional, por lo tanto, NO se utilizaran llaves para realizar la apertura, es por ello que se implementa dentro de la casa un sensor ultrasónico a una altura superior a los $1.80 \mathrm{mtrs}$. El cual recibirá una señal a una distancia que permita identificar que una persona adulta es la que realiza la operación de apertura desde el interior de la vivienda; no bastará con colocar la mano al frente o algún objeto para activar el actuador.

Los botones serán colocados en dos puntos estratégicos en la puerta y en el marco de la puerta, con el objetivo de estar lo mas ocultos posible, pero que realicen la acción adecuadamente, ambos tienen la tarea de confirmar el estado de la puerta ya sea cerrada o abierta, con esto se busca tener la confirmación adecuada y evitar que alguien juegue con los botones y pueda engañar al sistema, ya que del estado de los botones (presionados o NO presionados) será la acción que realice el servomotor y la activación de la alarma. Para la conexión, se utiliza una ESP8266, el cual esta configurado para obtener una dirección IP de un Punto de Accesos a Internet, con lo cual se tendrá conectividad con Firebase la base de datos en tiempo real que mantendrá a la mano la configuración de todo el sistema y es el punto de encuentro entre todas las partes del sistema.
En la figura 3 se muestra un fragmento del diagrama de conexión de los dispositivos con el controlador.

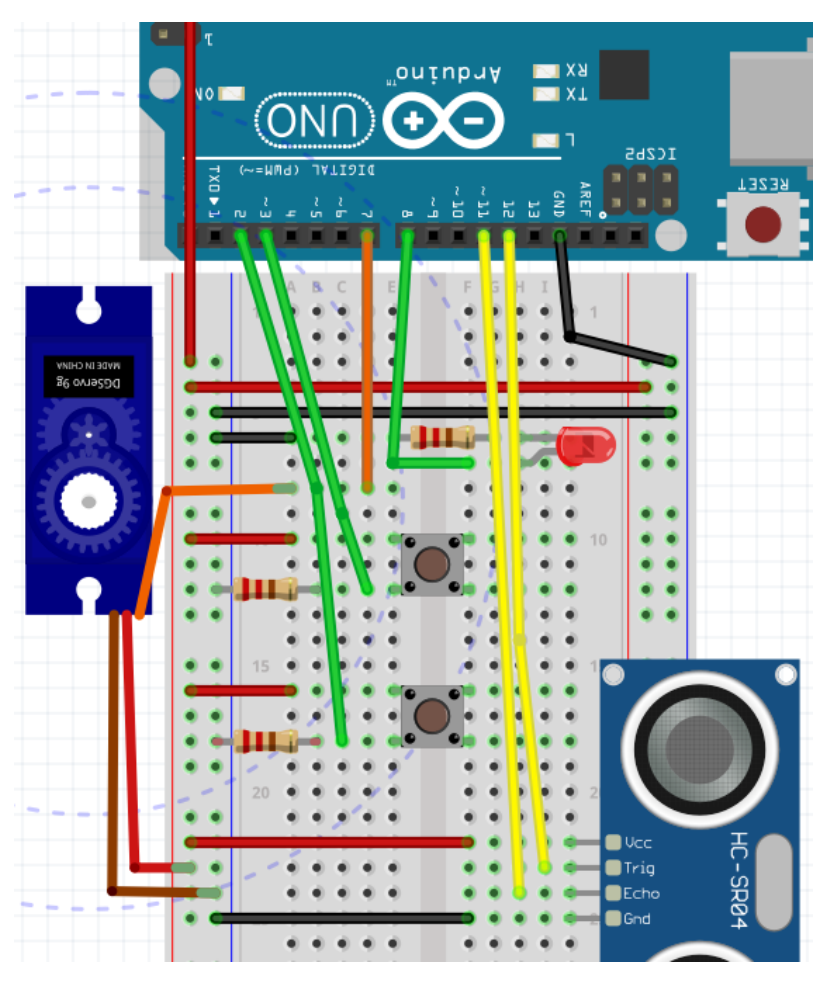

Figura 3 Parte del Diagrama de Conexión de Componentes

Programación.

La programación de Arduino se realiza con Node.js que se basa en JavaScript, el cual maneja una estructura simple para el desarrollo y permite concentrar gran parte de una solución en una misma interface de desarrollo, así como manejar un estándar en el lenguaje de programación. De primera instancia se requiere importar algunas librerías para el manejo de los sockets de conexión para lo cual se realizan algunas declaraciones, como se muestran en la Figura 4.

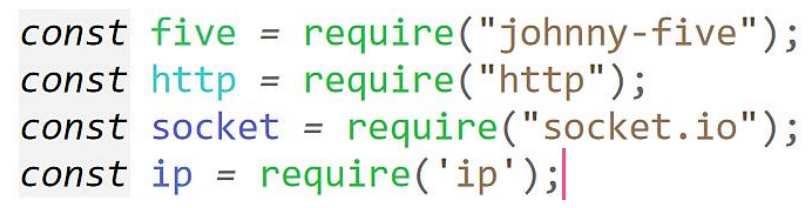

Figura 4 Importación de Librerías

Para realizar la interacción entre la unidad de control (Arduino) y las plataformas de visualización, ya sea web o aplicación móvil, es necesario utilizar un protocolo, en este caso se utiliza el HTTP, el cual trabaja con un servidor web que escucha el puerto 8080, el cual se crea, como lo indica la figura 5. 
// server

const app $=$ http. $\operatorname{createServer}() \cdot$. listen $(8080)$; const io $=$ socket.listen $(\mathrm{app})$;

Figura 5 Configuración del servidor web

El método (sockets) esta a la escucha de eventos en el servidor de manera local, de igual manera es capaz de emitir un nuevo evento que escuchara un dispositivo móvil. Código en la figura 6

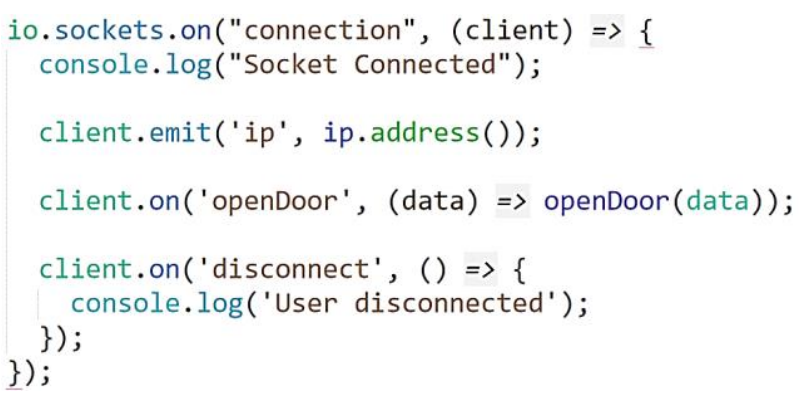

Figura 6 Método sockets

Dentro de la programación del dispositivo, se realizan los métodos necesarios para la verificación del estado de la puerta, pero también se realiza codificación para realizar acciones con los actuadores, en la Figura 7 se muestra el código utilizado para abrir la puerta, este método esta implementado con JavaScript en Node.js.

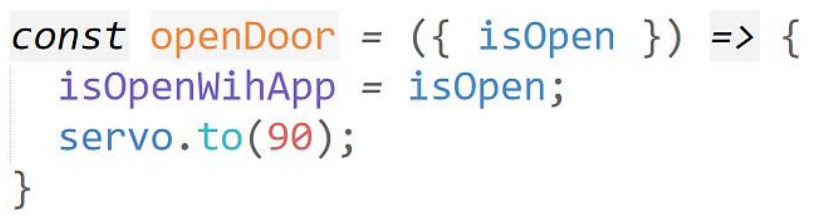

Figura 7 Código Implementado para abrir la Puerta

Este sistema tiene como prioridad la revisión constante de la puerta, conocer el estado actual de la misma, así como identificar cuales son las acciones realizadas para la apertura de la puerta. Esta verificación se implementa con ayuda de los botones colocados en la puerta, los cuales envían una señal al controlador cuando la puerta está abierta. El controlador considera que la apertura de la puerta está bajo control cuando previamente se recibe una señal desde la aplicación móvil o página $\mathrm{Web}$, en caso de NO recibir esta señal previa, el sistema indicara una operación invalida y notificara a las aplicaciones. Esta acción es la razón de ser del sistema de seguridad, ya que recibir la señal de apertura desde un dispositivo valido, permite $\mathrm{NO}$ activar las notificaciones (Alarmas).
En la figura 8 se muestra un fragmento del código implementado.

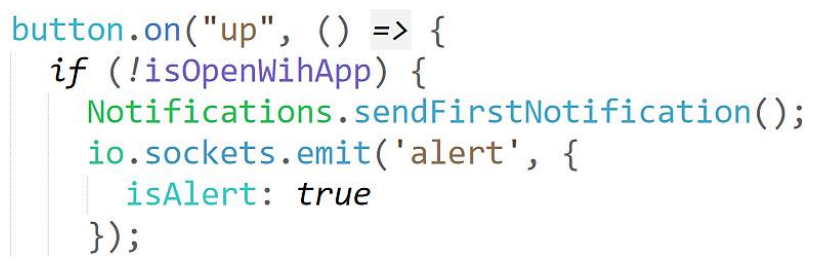

Figura 8 Código de notificación de puerta abierta

Una de las características adicionales de este sistema, es que implementa la interacción de manera local y de manera remota, es decir permite interactuar con un dispositivo móvil en la red LAN o Wireless LAN sin necesidad de internet, para ello se implementa el código de la figura 9 .

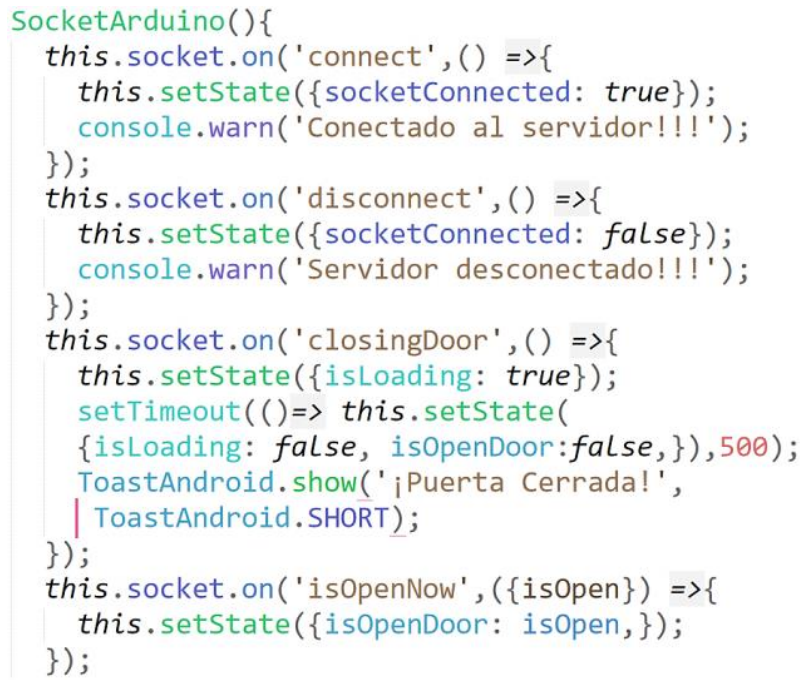

Figura 9 Código de Interacción en la red local

Por otro lado, la aplicación móvil también debe poder interactuar con el sistema de manera remota, esto para recibir notificaciones fuera de casa y donde quiera que se tenga acceso a una conexión de datos $3 \mathrm{G} \mathrm{o}$ 4G, o quizá en el trabajo con una conexión WiFi en una red distinta a la de casa. Para ello se implementa una base de datos con Firebase, la cual esta disponible en la nube a través de una cuenta de Google. En la imagen 10 se muestra parte del código implementado para que el controlador interactúe con la aplicación móvil por medio de Firebase.

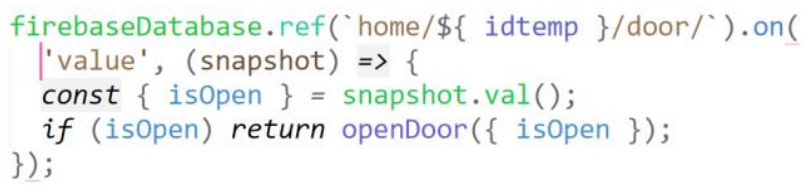

Figura 10 Código para revisar Firebase 
Considerando que la aplicación móvil puede estar instalada en varios dispositivos, asi como la posibilidad de tener varios usuarios que tienen derecho a utilizar la aplicación, es que se requiere implementar un historial de eventos, el cual permite dar un seguimiento a todos los eventos que genera la puerta. En la Figura 11, se muestra el código utilizado para la generación del historial desde la aplicación móvil.

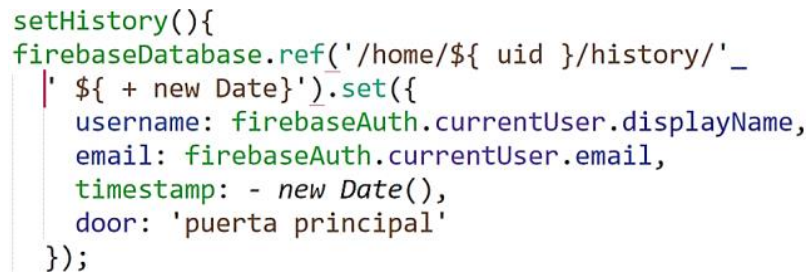

Figura 1110Código para crear historial de eventos

Aplicación Web de Gestión que se implemento para este proyecto es realizada en electrón y con la combinación de herramientas Javascript, en la figura 12 se muestran fragmentos de código para realizar la importación de dependencias de electrón, importación del modulo de Arduino y generación del índex de la aplicación de escritorio.

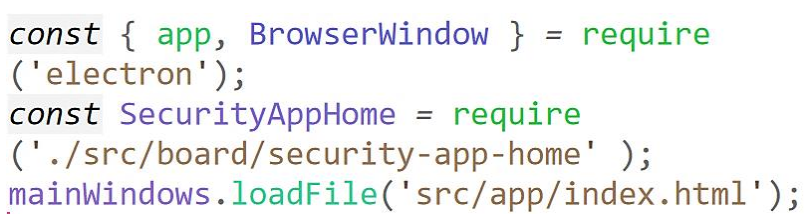

Figura 12 Código de creación de aplicación de escritorio fragmento

La arquitectura implementada en este proyecto se basa en el modelo Cliente Servidor, en este caso la aplicación móvil constituirá la parte cliente, y la base de datos será alojada en el servidor de Firebase al que se accede mediante el uso de su API.

El cliente es quien inicia las solicitudes, teniendo un papel activo en la comunicación, y espera la respuesta del servidor, el cliente interactúa con el usuario a través de una interface gráfica, por otro lado el receptor de la solicitud, es decir el servidor espera a que lleguen solicitudes de los clientes, tras la recepción de la solicitud se procesa la información, se almacena y se envía una copia al cliente.

\section{Resultados}

Como prototipo final, se obtuvieron varios elementos que forman parte del sistema, una aplicación WEB, una aplicación móvil, una base de datos en Firebase y un circuito electrónico que interactúa con cada uno de los elementos, con ello el usuario tendrá la posibilidad de monitorear el acceso a su hogar mediante una aplicación de escritorio que estará en el servidor local, lo cual le permitirá incluso gestionar a todos los usuarios que tienen acceso. En la figura 13 se muestra la pantalla principal de la aplicación móvil, la cual tiene como pantalla principal un botón para abrir la puerta configurada para este fin, se considera un PIN o código de seguridad para que ningún usuario que encuentre el dispositivo pueda hacer mal uso del sistema.

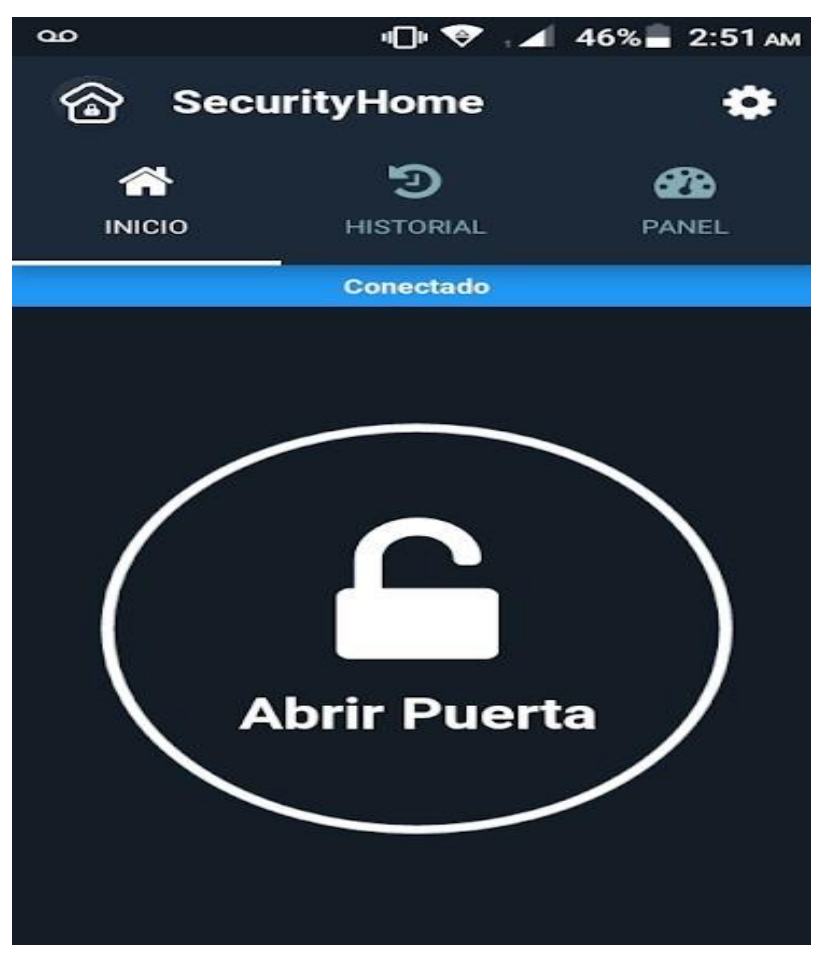

Figura 13 Pantalla principal Aplicación Móvil

Por otro lado, se tiene la aplicación de escritorio, la cual permite realizar acciones adicionales como la creación de usuarios y códigos de seguridad, la baja de usuarios, así como la modificación de los mismos. Entre otras acciones se destaca el historial de eventos, el cual permite dar un seguimiento de fechas y horarios en los que ha ocurrido la apertura. En la Figura 14 se muestra la pantalla del historial de la aplicación de escritorio. 


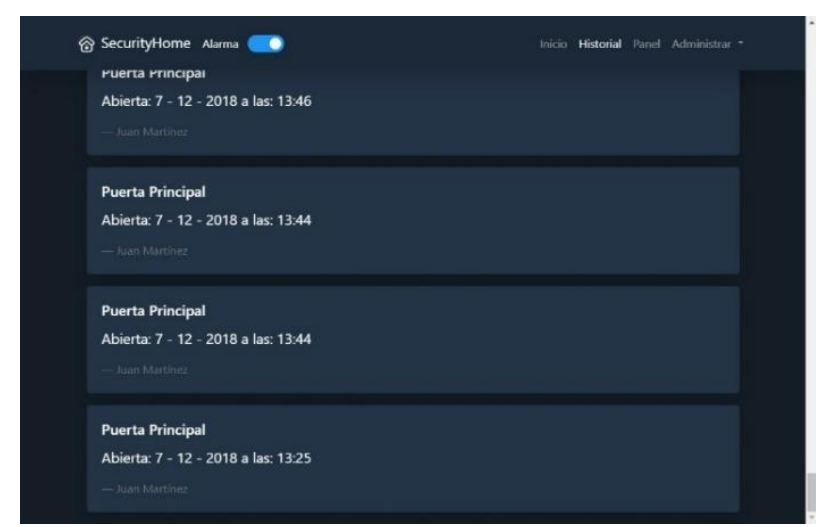

Figura 14 Historial de eventos en Aplicación de Escritorio

En la aplicación de escritorio, se tiene la posibilidad de Activar o desactivar la alarma, esta opción solo desactiva la alarma sonora, el resto de funciones del sistema continúan activas. Cuando la puerta se abre sin previa autorización de la aplicación móvil o de la aplicación de escritorio, se envían mensajes a las dos aplicaciones y en su caso se activa una alarma sonora que permite darnos cuenta del suceso en tiempo real, la aplicación móvil da la oportunidad de realizar una llamada a algún numero de emergencia, pero si se tiene controlado el sistema, entonces se puede declinar de llamar a la policía. En la figura15 se muestra el mensaje o notificación de alerta.

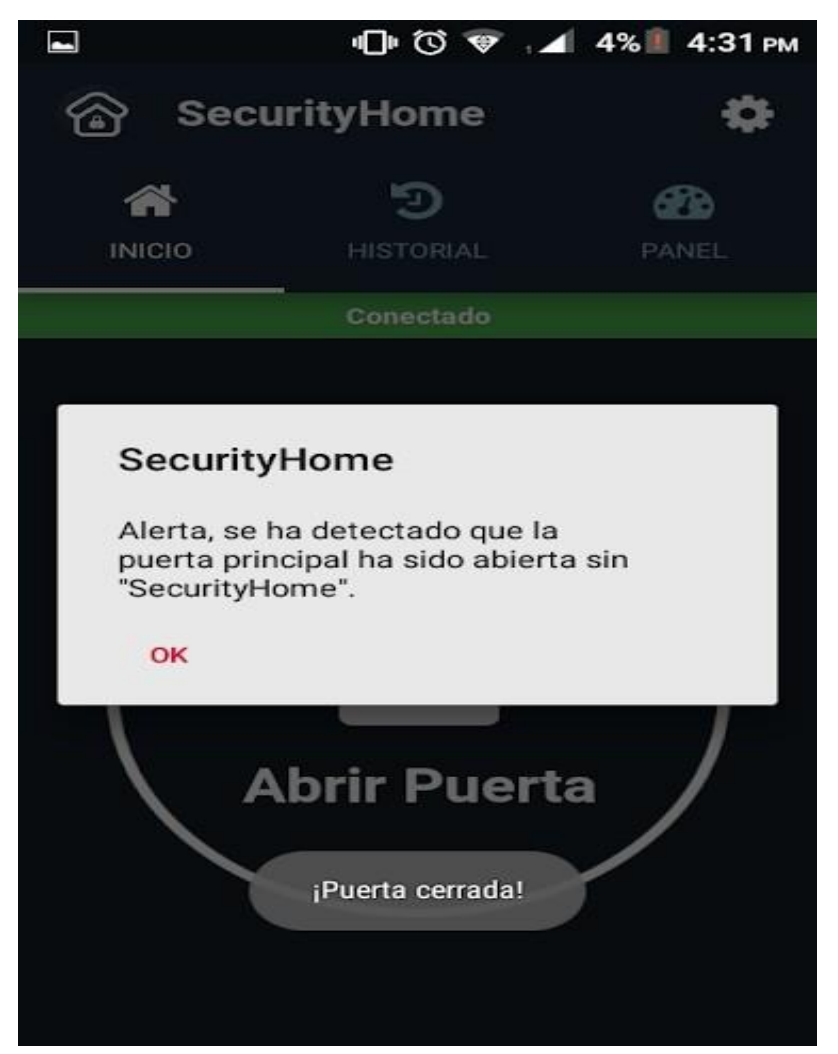

Figura 15 Alerta de apertura de puerta en móvil
En cuanto al funcionamiento de la puerta, las pruebas básicas se realizaron en una maqueta la cual tiene el mismo funcionamiento de una puerta de casa.

Este proyecto se genero como una alternativa a los sistemas de alarma que ya implementa Internet de las Cosas, como parte de los resultados es la participación del equipo de desarrollo en el evento denominado "Global Fest Universitario 2019" que organiza el H. ayuntamiento de Tehuacán" en el estado de puebla, participando en la categoría de Innovación, obteniendo el ler lugar en la primera eliminatoria y el 2do lugar en la gran final del evento.

En esta competencia se generó el modelo de negocio para la fabricación, promoción, venta e instalación de un producto, el cual los usuarios interesados en adquirirlo pueden solicitar los servicios del equipo de trabajo para realizar la adquisición del equipo, instalación del mismo y el soporte para el correcto funcionamiento del sistema.

En la Figura 16, se muestra la presentación realizada en la primera etapa de evento.

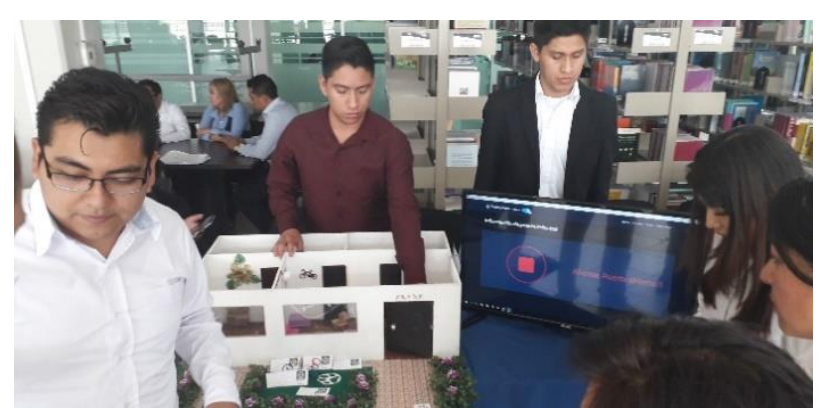

Figura 16 Equipo de trabajo en el Globalfest primera Etapa

\section{Agradecimientos}

Por su brillante participación en el desarrollo de este proyecto a Juan Martínez Osorio, Félix Alberto García Martínez, Juan Carlos Sánchez Pacheco, Miguel Adolfo Correa, Jorge Luis Fernando Martínez Hernández y Abiram Arana Gómez. El presente documento refleja de manera resumida el esfuerzo que ustedes, el equipo de trabajo ha realizado para culminar la primera etapa de este proyecto. 


\section{Conclusiones}

La implementación del sistema de seguridad tiene su origen a la inquietud de darle alternativas al problema del hurto que sufren las personas en sus hogares; ya que la ciudadanía no pude estar tranquila pensando que en cualquier momento personas no autorizadas puedan entrar a sus hogares. considerando por el hecho de ser un problema común en la ciudad de Tehuacán, toma mayor importancia para el equipo de trabajo. El sistema de seguridad pretende aumentar la seguridad de los hogares de sus usuarios, de una manera fácil, permitiendo al usuario poder gestionar y controlar el acceso a sus hogares desde su celular sin la necesidad de utilizar llaves o algún otro método de seguridad con cerraduras tradicionales. De esta manera el acceso a puertas principales al hogar, puerta del garaje, puerta de emergencia, de la oficina, y cualquier otro tipo de acceso podrá ser fácilmente controlado por el usuario desde su dispositivo móvil gracias a el sistema de seguridad de Internet de las cosas.

El sistema funcionará gracias a una aplicación que deberá estar instalada en un teléfono inteligente, que a su vez tendrá una conexión con los dispositivos que estarán instalados en las puertas, de esta manera solo el usuario de la aplicación tendrá el poder de abrir sus puertas de forma segura.

Este sistema pone a la mano del usuario la posibilidad de llamar a seguridad publica para atrapar a los ladrones en fragancia, y de esta manera evitar el robo de casa habitación, la alarma sonara en la aplicación de escritorio y en el dispositivo móvil, así como la generación de notificar al usuario en tiempo real.

El proyecto inicial esta terminado al cien por ciento, sin embargo, a partir de la evaluación en el concurso GlobalFest Universitario, surgen propuestas e ideas que los mismo evaluadores y personas que preguntan sobre el proyecto han querido aportar desde su experiencia. Es por ello que se ha considerado mejorar el proyecto para controlar mas puertas, no solo la principal, controlar las ventanas y mantener un sistema de tradicional de chapas para puerta, pero mejoradas para esta aplicación, entre otros aspectos.
Así mismo, se han recibido propuestas en cuanto al modelo de negocio establecido, el cual consiste en la venta de un producto, sin embargo, al tener un costo relativamente alto, se considera bajar los costos para tener un esquema de renta o alquiler.Definitivamente falta mucho que mejorar en este proyecto, pero es satisfactorio el resultado obtenido en la aplicación del Internet de las cosas, es un paso más con respecto al proyecto reportado con el articulo "Aplicación practica de internet de las cosas" en el año 2018, el cual sirve como antecedente sobre este trabajo.

\section{Referencias}

330ohms. (22 de Febrero de 2019). ¿Qué son los actuadores electrónicos? Obtenido de https://blog.330ohms.com:

https://blog.330ohms.com/2013/10/14/que-sonlos-actuadores-electronicos/

Abernethy, M. (02 de 05 de 2019). ¿Simplemente qué es Node.js? Obtenido de https://www.ibm.com:

https://www.ibm.com/developerworks/ssa/open source/library/os-nodejs/index.html

Arduino. (25 de febrero de 2019). https://www.arduino.cc. Obtenido de Arduino: https://www.arduino.cc/en/Guide/Introduction

Caballero, J. G. (14 de marzo de 19). DevCode.la. Obtenido de ¿Cómo funciona React.js?: https://devcode.la/blog/comofunciona-reactjs/

Caballero, J. G. (18 de marzo de 2019). ¿Qué es JavaScript? Obtenido de Devecode: https://devcode.la/blog/que-es-javascript/

CampusMVP. (10 de marzo de 2019). https://www.campusmvp.es. Obtenido de Las 5 principales ventajas de usar Angular para crear aplicaciones web: https://www.campusmvp.es/recursos/post/las-5principales-ventajas-de-usar-angular-para-crearaplicaciones-web.aspx

Castro, M. (20 de mayo de 2019). Construyendo aplicaciones web de escritorio con Electron. Obtenido de https://medium.com: https://medium.com/@BlackHarpy/construyend o-aplicaciones-web-de-escritorio-con-electron974c3e2174b 
Decena, E. (20 de abril de 2019). Servidor real time con socket.io. Obtenido de https://medium.com:

https://medium.com/@eddydecena/servidorreal-time-con-socket-io-18e84d39d12b

Developers, G. (09 de abril de 2019). Firebase Realtime Database. Obtenido de https://firebase.google.com:

https://firebase.google.com/docs/database/?hl=e s-419

Ecured. (2019 de marzo de 19). Lenguaje de programación (informatica). Obtenido de https://www.ecured.cu:

https://www.ecured.cu/Lenguaje_de_programac i\%C3\%B3n_(inform\%C3\%A1tica)

Foundation., N. J. (06 de enero de 2019). Node js. Obtenido de https://nodejs.org/es/

Gil, S. V. (16 de agosto de 2019). Se duplican los robos en casas. El Mundo, págs. https://www.diarioelmundo.com.mx/index.php/ 2019/04/26/se-duplican-los-robos-en-casas/.

González, V. R. (20 de febrero de 2019). http://platea.pntic.mec.es. Obtenido de Estructura de un robot industrial: http://platea.pntic.mec.es/vgonzale/cyr_0204/ctr 1_rob/robotica/sistema/morfologia.htm\#controla dor

LATAM, M. (20 de febrero de 2019). Mecatronica LATAM. Obtenido de https://www.mecatronicalatam.com:

https://www.mecatronicalatam.com/tutorial/es/s ensores

MUEDANO, M. (16 de Agosto de 2019). Faltan a México 96 mil policías; incumple estándar de la ONU. Excelsior, págs. https://www.excelsior.com.mx/nacional/faltana-mexico-96-mil-policias-incumple-estandarde-la-onu/1262031.

Rosas, F. (16 de Agosto de 2019). México con suficientes policías de acuerdo con estándar de ONU. EL Heraldo de México, págs. https://heraldodemexico.com.mx/opinion/mexic o-con-suficientes-policias-de-acuerdo-conestandar-de-onu-\%EF\%BB\%BF/.
Rouse, M. (27 de febrero de 2019). Internet de las cosas (IoT). Obtenido de https://searchdatacenter.techtarget.com:

https://searchdatacenter.techtarget.com/es/defin icion/Internet-de-las-cosas-IoT

S.A.C., C. T. (16 de febrero de 2019). https://gruponavarro.pe. Obtenido de Todo sobre los Sistemas de Seguridad Electrónica: https://gruponavarro.pe/sistemas-seguridad/quees-la-seguridad-electronica/

SÁNCHEZ, O., NOGUERON, A., CURIOCA, Y., \& EXY, R. (2018). Aplicación práctica de internet de las cosas . Revista de simulación computacional, 30-35.

SEGURIDAD, N. (15 de febrero de 2019). ¿CÓMO FUNCIONA UN SISTEMA DE SEGURIDAD? Obtenido de http://ncseguridad.es:

http://ncseguridad.es/2017/06/12/comofunciona-un-sistema-de-seguridad/

Signal, O. (20 de abril de 2019). Product Overview. Obtenido de onesignal.com: https://documentation.onesignal.com/docs

tdrobotica.co. (25 de febrero de 2019). Conectividad en el Internet de las Cosas. Obtenido de http://aprender.tdrobotica.co: http://aprender.tdrobotica.co/cursos/conectivida d-en-el-internet-de-las-cosas/lessons/mirandomas-lejos/

Valdez, A. (16 de agosto de 2019). Ofrecen consejos para evitar robos a casa. El Mundo, págs.

https://www.diarioelmundo.com.mx/index.php/ 2019/07/18/ofrecen-consejos-para-evitar-robosa-casa/. 